\title{
Histogram Equalization Tool: Brightness Preservation and Contrast Enhancement using Segmentation with Opening-by-Reconstruction
}

\author{
Ramandeep Kaur \\ Assistant Professor \\ DAV College, Jalandhar, India
}

\begin{abstract}
Sundry improvement plans are used for improving a picture which incorporates ash scale control, sifting and Histogram Equalization (HE). The issue with pictures is that, their quality depends upon a number of different variables like lighting in the picture catching area, commotion and capability of the administrator. The writing addresses the verbalized issue widely and presents answers for them. Contrast improvement systems are used for correcting visual nature of low difference pictures. Histogram Equalization (HE) is one such procedure used for difference upgrade. The proposed illustrations have a few shared traits in their procedures. Approximately every one of them is at fluctuation either in histogram leveling strategies or in picture quality estimation instruments. An instrument is lost in the writing that is proficient to improve the picture and even perform the examination. In this paper, a GUI apparatus is composed which is coupled with different procedures of picture improvement through histogram balance. Opening-byrecreation is a standout amongst the most effective picture division strategy is used to attain to the craved results. To assess the adequacy of the delineated systems; PSNR, Tenengrad, and Absolute Mean Brightness mistake (AMBE) are used as parameters. The results are decently backed by the parameter estimations toward the end.
\end{abstract}

\section{General Terms}

Digital Image Processing, Image Enhancement and Histogram Equalization.

\section{Keywords}

Contrast Enhancement, Brightness Preservation, Foreground Enhancement, Histogram Equalization, Quality Measures, Cumulative Density Function.

\section{INTRODUCTION}

Digital Image Enhancement is a standout amongst the most significant picture preparing innovation, which is key to enhance the visual appearance of the picture or to give a more dominant change representation for future robotized picture handling, for example, picture examination, Processing, location, division and apperception [1]. Contrast enhancement is a considerable region in picture handling for both human and machine vision. It is generally used for therapeutic picture transforming and as a preprocessing venture in verbalization perception, composition amalgamation, and numerous other picture/feature handling applications [2-5]. A sample of the upgrade is demonstrated in the accompanying Fig. 1 in which, when we augment the differentiation of a picture and channel it to extract the commotion; the picture looks much better. Enhancement is a subjective zone of picture handling. Improvement in the nature of these corrupted pictures can be attained to by using the application of the upgrade procedure.
Distorted Image

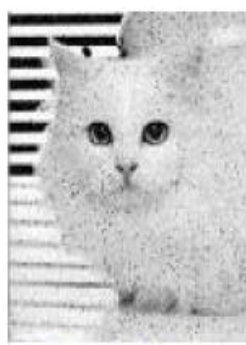

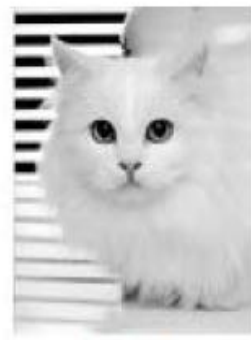

Enhanced Image
Figure 1 Image Enhancement

Numerous pictures have low a dynamic scope of the force values because of lacking light and thusly need to be transformed up to being displayed. Sizable voluminous number of procedures has focused on the upgrade of light black level pictures in the spatial area. Complexity is caused by the distinction in luminance reflectance from two nearby regions. In our visual observation, complexity is unfaltering by the distinction in the shade and brightness of an article with different items. On the off chance that the picture complexity is exceedingly focused on an absolute range, the data may be bewildered in those ranges which are excessive and consistently throughout. The issue is the contrast enhancement of a picture with a specific end goal to speak to all the data in the information picture. Brightness preservation methods are popular to the shopper electronic items. The histogram equalization (HE) based luminosity protecting techniques grade to induce undesirable relics in [6].

Despite fundamental point of interest in histogram adjustment, it has an important downside of transmuting the brilliance all around, which prompts either under-immersion or overimmersion of the central areas. Because of this thinking, for the execution of difference upgrade in purchaser electronic items it is viewed as that the loss of force values by the histogram handling ought to be lessened in the yield picture. The initially changed histogram challenge has been proposed by Kim, in 1997 [7] using bi-histogram balance (BHE) strategy. In this paper, a GUI device is composed which is focused around histogram equalization methods. These strategies are talked about and investigated. Few systems from every class are tried using diverse test pictures and looked at, predicated on picture quality appraisal qualities. This paper is organized in five sections. Section 1 distributes brief introduction, Section 2 discusses the chosen methodology. Section 3 covers various available methods cognate to histogram equalization, which preserves the effulgence as well as the contrast of the image. Section 4 describes different image quality assessment methods, comparison of the above stated methods is given in Section 5 and Section 6 provides concluding remarks. 


\subsection{Histogram Equalization}

HE procedures are broadly used in our quotidian life, such that in the field of Medical picture transforming, shopper gadgets, picture matching and testing, verbalization perception and surface blend on the grounds that it has high productivity and straightforwardness. The best well known strategy for differentiation upgrade of pictures is histogram balance (HE) [7-11]. It is one of the decently kenned techniques for upgrading the complexity of a given picture as per the examples circulation [12, 13]. HE is a basic and effective complexity improvement strategy which conveys pixel values consistently such that upgraded picture have direct aggregate histograms. It develops the difference of the high histogram districts and clamps the differentiation of the low histogram areas [14]. The HE procedure is a worldwide operation, consequently; it doesn't save the picture luminosity. $\mathrm{HE}$ has been generally connected when the picture needs an upgrade, for example, radar picture preparing, therapeutic picture handling, surface amalgamation, and verbalization perception $[10,15-16]$. HE routinely brings two sorts of relics into the balanced picture to be specific over-improvement of the picture districts with more successive light black levels, and the loss of differentiation in the picture areas with less continuous ash levels [17]. To surmount these disadvantages a few HE-predicated procedures are proposed and are more focused on the safeguarding of picture brightness than the enhancement of picture complexity. Few techniques frequently cause pictures with angering visual ancient rarities and unnatural appearances; however the picture brilliance is safeguarded to some degree [18].

Consider the discrete grayscale info Image $X=x(i, j)$, having $\mathrm{L}$ discrete levels, where $\mathrm{x}(\mathrm{i}, \mathrm{j})$ speaks to the power levels of the picture in the spatial area ( $\mathrm{i}, \mathrm{j})$. Let histogram of Image $\mathrm{X} \mathrm{H}(\mathrm{x})$. Presently the likelihood thickness capacity $\operatorname{pdf}(\mathrm{x})$ can be characterized as [1]-

$\operatorname{pdf}(\mathrm{Xk})$ or $\mathrm{p}(\mathrm{Xk})=\mathrm{nk} / \mathrm{N}, \quad$ where $0 \leq \mathrm{K} \leq(\mathrm{L}-1)$

$\mathrm{L}$ is the aggregate number of gray levels in the image,

$\mathrm{N}$ is the Total number of pixels in the image,

$\mathrm{nK}$ is the aggregate number of pixels with the same intensity level K

From the $\operatorname{pdf}(X)$ (1) the cumulative distribution function $\operatorname{cdf}(\mathrm{Xi})$ is defined as-

$\operatorname{cdf}(\mathrm{Xi})$ or $\mathrm{c}(\mathrm{Xi})=\sum_{\mathrm{i}=0}^{\mathrm{K}} \mathrm{p}\left(\mathrm{X}_{\mathrm{i}}\right)$

Note that $\operatorname{cdf}(\mathrm{XL}-1)=1$ from eq (1) and (2)

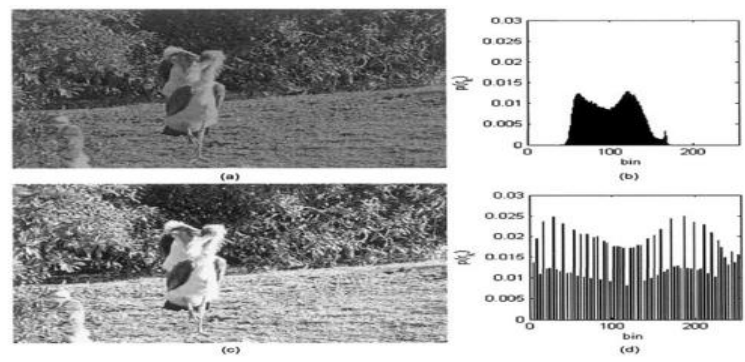

Figure 2 Histogram Equalization a) Histogram b) Equalized histogram

Histogram equalization is a plan that maps the info picture into the whole dynamic range by utilizing the total dispersion work as a change capacity [x0, XL-1] by utilizing the aggregate conveyance work as a change capacity. We should characterize the change capacity $\mathrm{f}(\mathrm{x})$ utilizing total dispersion capacity cdf (Xi) [1] as-

$\mathrm{f}(\mathrm{X})=\mathrm{X}_{0}+\left(\mathrm{X}_{\mathrm{L}-1}-\mathrm{X}_{0}\right) \mathrm{x} \operatorname{cdf}\left(\mathrm{X}_{\mathrm{i}}\right)$

Then the output image of histogram equalization, $\mathrm{Y}=\mathrm{yi}$ can be expressed as

$$
\begin{aligned}
& Y=f(X) \\
& Y=\{f(x(i, j)) \mid \forall x(i, j) \in X\}
\end{aligned}
$$

The above portray the histogram equalization on grey scale picture. Be that as it may it can also be utilized on shade picture by applying the same technique discretely to the Red, Green and Blue Component of the RGB color picture.

\section{HISTOGRAM EQUALIZATION BASED TECHNIQUES}

Histogram adjustment is a straightforward and an effectual contrast enhancement method which appropriates pixel values consistently such that improved picture have cumulative linear histogram and is a worldwide operation. In this way, it doesn't protect the picture radiance. To surmount these blemishes and augmentation contrast improvement and brightness preserving numerous HE-predicated strategies have been proposed.

\subsection{Bi-Histogram Equalization Methods}

Histogram equalization techniques partition the histogram into two sub-histograms predicated on diverse isolating focuses. After that, each one sub-histogram is evened out independently predicated on histogram balance. At the point when contrasted with Histogram Equalization technique, these strategies can safeguard picture brightness mode.
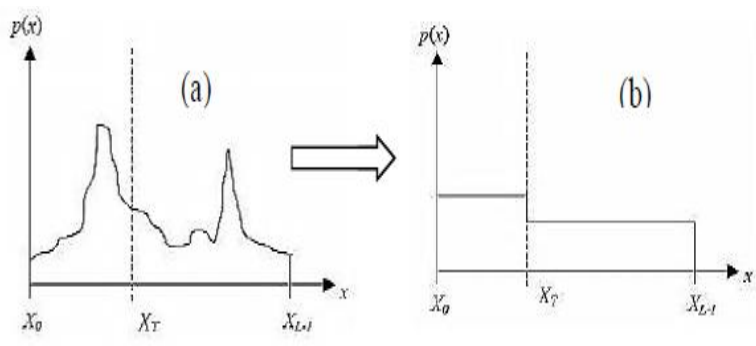

Figure 3 Bi-histogram Equalization Method

a) Input Histogram Divides into Two sub-histograms (b) Equalized Sub-histograms

To begin with Brightness Preserving Bi-Histogram Equalization (BBHE) has been proposed by Kim in 1997 [7] to safeguard the mean luster of a given picture while difference is upgraded and it protects the radiance of pictures at some degree and indicated more dominant result than HE. Much the same as BBHE, Wang et al., In 1999 [19] proposed Dualistic Sub-Image Histogram Equalization (DSIHE), yet this system utilized average esteem as a part of lieu of intend to separate the info histogram and indicated more prevalent brightness protecting than BBHE and HE. DSIHE is the best preparing method to save the perfect picture blaze and furthermore upgrade the picture data usefully. BBHE and DSIHE are very less compatible for pictures obliging higher level of brightness safeguarding to dodge vexing antiques.

For higher level of conservation Chen and Ramli in 2003 [20, 21] proposed Minimum Mean Brightness Error Bi-Histogram Equalization (MMBEBHE) a prolonged strategy for BBHE and the divergence predicated on edge level, which yields 
least Absolute Mean splendor Error (AMBE). A definitive objective abaft this strategy is to authorize the most extreme level of brilliance conservation in Bi-Histogram Equalization to sidestep obnoxious relics and unnatural improvement because of excessive leveling and withal to figure an effective, recursive and whole number predicated answer for rough the yield mean as a capacity of limit level. Mimicked results from [22], MMBEBHE serenely be tokens that it has safeguarded more prevalent brilliance and yielded a more common improvement. BBHE and MMBEBHE have a more prevalent safeguarding and upgrade levels contrasted with $\mathrm{HE}$ and DSIHE. Although, MMBEBHE shows poor brightness conservation and improvement, where the pictures that require significantly more brilliance safeguarding it neglects to control the over enhancement of the picture.

Bi-Histogram Equalization with Neighborhood Metric (BHENM) has been presented by Sengee et al., [23] in 2010, which isolate the cosmically gigantic histogram canisters that because washout antiques into sub-receptacles using neighborhood measurements, in addition to the histogram of the flawless picture is separated into two sub-histograms predicated on the mean of the histogram. At that point, subhistograms are balanced freely using refined histogram leveling, which induce compliment histograms. The area qualification metric is to sort pixels of equivalent force into diverse sub-canisters, to change picture nearby difference, and to dissever the histogram into two sub-histograms and after that adjust them autonomously to safeguard the picture brightness.

Zuo et al., proposed Range Circumscribed Bi-Histogram Equalization (RLBHE), in 2012 [24], which partitions the information, histogram into two free sub-histograms by a limit that minimizes the intra-class change. This was completed to adequately dissever the items from the foundation. This system accomplishes outwardly an all the more satisfying differentiation upgrades while keeping up the data blazes and it is effortless to actualize in legitimate time transforming.

\subsection{Multi-Histogram Equalization Methods}

To improve picture contrast, preserving brightness and to enhance the characteristic looking of the pictures, multihistogram adjustment method deteriorates the info picture into a few sub-images, and after that applies the traditional histogram equalization procedure to each of the subhistogram. Picture handled by Multi-HE routines save the picture brilliance and deflect exordium of undesirable relics yet not altogether improve the differentiation [32].

Wongsritong et al., [25], has proposed Multi Peak Histogram Equalization with Brightness Preserving (MPHEBP) in 1998, to alter the luster protecting of the picture. In this execution, the data histogram will be smoothed and separated predicated on the neighborhood maxima. Wongsritong et al. asserted that the execution of MPHEBP in keeping up the mean luminosity is more dominant than BBHE.

Recursive Mean-Separate Histogram Equalization (RMSHE) has been proposed by Chen and Ramli in 2003 [26], they picked to separate the data histogram into two, predicated on its mean in advance of leveling them freely. This divergence is carried out one time in BBHE, yet in this technique early histogram (divided) were further partitioned predicated on their mean worth. It has been investigated scientifically that the yield picture's mean brightness would meet to the data picture's mean radiance as the quantity of recursive mean disunities increments. So as to attain to higher luster conservation, this model is proposed to perform the meanest partition recursively and separate the ensuing histograms again predicated on their individual assigns. Recursive SubImage Histogram Equalization (RSIHE) like RMSHE is proposed by Sim et al., [27] in 2007 and it separates the info, histogram predicated on an ash level with average, RMSHE make utilization of mean-partition and both the systems have the same qualities in evening out the sub-histograms. Both systems demonstrated great blaze, saving due to multi separation of histogram; however, for bright images these techniques lead to over improvement.
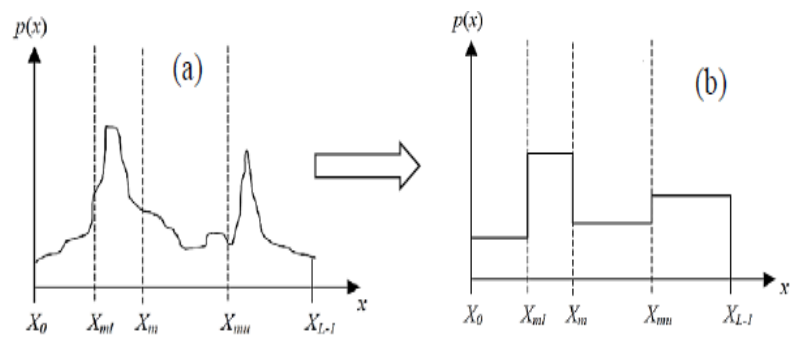

Figure 4 Recursive Mean-Separate Histogram Equalization (RMSHE) a) Separated Histogram (b) Equalized Histogram $(r=2)$

Dynamic Histogram Equalization (DHE) has been exhibited by Wadud et al., [28] in 2007 to take out the ascendance of higher histogram sections on lower histogram parts in the picture histogram and to control the measure of stretching out of light dark levels for possible change of the picture offers by utilizing close-by minima disseverment of the histogram. DHE has showed more prevailing and a smooth update of the picture. Of course, the DHE rejects the mean iridescence sparing and slants to drive submersion doodads. To surmount the drawback of the DHE, Brightness Preserving Dynamic Histogram Equalization (BPDHE) have been proposed by Ibrahim and Kong [29] in 2007, an incremental strategy for the DHE and Multi Peak Histogram Equalization with Brightness Preserving (MPHEBP) and parcels the data histogram predicated on adjacent most noteworthy quality. BPDHE shows more predominant contrast overhaul stood out from MPHEBP and mean quality, ensuring appeared differently in relation to DHE.

Menotti et al., [30] proposed Minimum Within-Class Variance Multi-Histogram Equalization (MWCVMHE) and Minimum Middle Level Squared Error Multi Histogram Equalization (MMLSEMHE) methodologies in 2007, to yield pictures with trademark appearances, to the detriment of many-sided quality change. MWCVMHE packages the data histogram into different sub-histograms by minimizing inside class contrast and after that applies histogram night out in every one sub-histogram discretely. MMLSEMHE utilizes the Otsu edge determination system to divid dissevering concentrates, up to adjusting every one sub-histogram unreservedly with HE. MMLSEMHE is more computationally multifaceted in light of the fact that it advises the perfect number of sub-histograms from all possible sub-histograms to minimize certain divergence limits [31]. Both the procedures, secure the glow to a more prominent degree, however the distinction change is less tight.

In 2008, Kim and Chung [18] proposed Recursively Separated and Weighted Histogram Equalization (RSWHE) to overhaul the picture separate and notwithstanding secure the picture shine. This methodology parts the information, histogram into two or more sub-histograms recursively predicated on the mean or normal of the picture. Withal they came to fruition sub-picture histograms will transmute through a weighting 
methodology predicated of the puissance law limit. RMSHE and RSIHE are homogeneous to RSWHE with respect to recursive histogram division; despite they don't execute the histogram weighting limit as in RSWHE. Both RSWHE-M and RSWHE-D are two different uses of RSWHE: that is, RSWHE-M performs the mean-based division and RSWHE-D performs the normal predicated division. The test outcomes show, the RSWHE-M framework is found to be more predominant than the RSWHE-D for brightness sparing and separation redesign.

Wadud et al., [32] in 2008, has been exhibited Spatially Controlled Histogram Equalization (SCHE) separating it to different sub-histograms until it discovers that no staggering fragment is shown in any of the from right off the bat made sub-histograms. After that a component powder level (GL) degree is relegated for every one sub-histogram to which its cinder levels can be mapped by histogram equalization. This entire element extent of light, dark levels of the yield picture is appropriated among the sub-histograms predicated on their component go in the data picture and aggregate scattering limit (CDF) of histogram qualities.

Sheet et al., [33] in 2010 acquainted an alteration with BPDHE strategy through fluffy insights of computerized pictures alluded to as Brightness Preserving Dynamic Fuzzy Histogram Equalization (BPDFHE) and it figures the fluffy histogram and afterward dividing the histogram into different sub-histograms predicated on neighborhood maxima. Each valley partition between two successive nearby maxima structures a segment. At the point when the element adjustment of these parts is performed, the peaks of the histogram don't get remapped and bringing about a more dominant safeguarding of the main picture - brightness while increasing the differentiation.

In 2012, Khan et al., [34] has proposed Weighted Average Multi Segment Histogram Equalization (WAMSHE), which partitions smoothed histogram into various portions predicated on ideal limits and leveled each one fragment by histogram evening out. WAMSHE demonstrated more prevalent luster protecting and difference improvement among Multi-histogram evening out strategies and moreover benefits to decrease the clamor show in the picture.

\subsection{Clipped Histogram Equalization Methods}

In general, histogram equalization contrasts the differentiation of the low histogram regions and grows the difference of the high histogram areas [14]. Accordingly, when the object of enthusiasm for a picture just involves a pinch of the picture, this article won't be prosperously upgraded by histogram balance and this system also colossally pushes the intensities towards the privilege or the left half of the histogram, creating level immersion impacts. To surmount these issues, Clipped Histogram Equalization (CHE) systems are used to confine the improvement rate. CHE reshape the data histogram by decreasing or increasing the worth in the histogram's canisters predicated on an edge limit up to the adjustment is occurring. This edge obligation is withal kenned as far as possible, or the level of the histogram. The histogram will be cut predicated on this edge esteem. Now and again cut part will be redistributed once more to the histogram and afterward histogram leveling is completed. Cut Histogram Equalization (CHE) is much a bigger number of more effectual for complexity upgrade than the subsisting HE-predicated
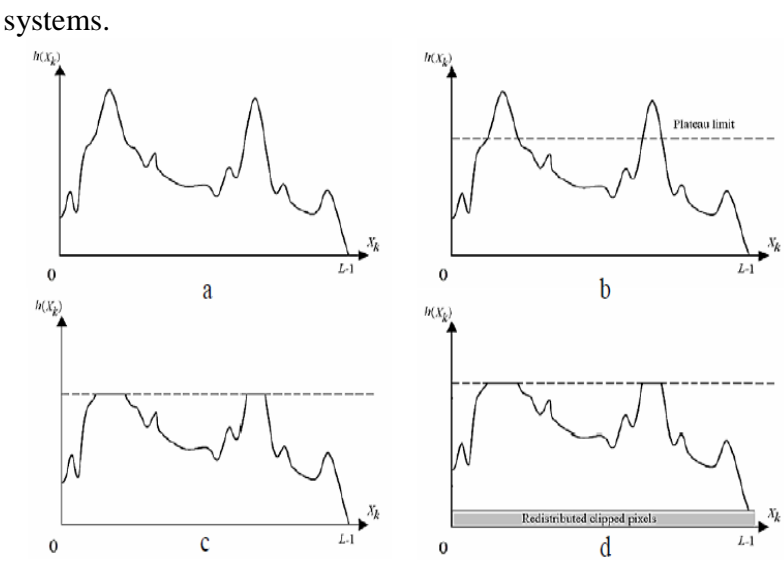

Figure 5 Clipped Histogram Equalization (CHE) Method a) The Original Input Histogram (b). The Settings of the Plateau Limit c) Clipping the Histogram based on the Plateau Limit (d). Redistribution of Clipped Portion Back into the Modified Histogram

The major shortcomings of CHE method are those methods that require manual setting of the plateau level of the histogram which are not opportune for automatic systems and some of the methods put weight to the modified histogram. The weight factor is additionally dependent to the utilizer.

Yang et al., [35] proposed a simple enhancement rate control mechanism Bin Underflow and Bin Overflow (BUBO) in 2003 that manages the rate of enhancement by putting constraints on the maximum and minimum gradient of the mapping function. The $\mathrm{HE}$ can revamps the contrast to variable rates, and additionally perform sundry image processing tasks such as ebony/white level stretch or automatic effulgence control with this enhancement rate control mechanism.

In 2006, Wang et al., [36] proposed Self-Adaptive Plateau Histogram Equalization (SAPHE) to enhance the main objects and suppress the background for infrared images. A histogram of the image is filtered in SAPHE with median filter to reduce the fluctuation and additionally to abstract some empty bins in the histogram and find the local maximum value and global maximum value of the histogram for plateau threshold value. By eradicating median filter from SAPHE, Nicholas et al., [37] introduced modified SAPHE (MSAPHE) in 2009 to enhance microscopic images. SAPHE failed to identify local peaks in the image and MASAPHE has overcome this issue.

Kim and Paik, in 2008 [15] presented a nascent differentiation upgrade strategy for controlling clamor enhancement and additionally safeguarding the perfect brilliance of the picture assigned as Gain-Controllable Clipped Histogram Equalization (GC-CHE). In the cutting level, picture histogram is trimmed and the cuts allotment is then recirculated to the whole dynamic range of regional standards directing the cutting increase. Amid the improvement of the complexity of a low light-level picture, the differentiation rise proportion is acclimated to understand the commotion enhancement difficulty as per the info picture and remunerate difference using the addition control strategy.

In 2009, Ooi et al., [38], proposed Bi-Histogram Equalization Plateau Limit (BHEPL) as the combination of the BBHE and cut histogram evening out. Related to BBHE, the BHEPL deteriorates the data picture into two sub-pictures by using the mean brightness of the picture. At that point, these subhistograms are cut by using as far as possible as the mean of 
the quantity of force event and the decayed sub-histograms are balanced freely. BHEPL strategy keeps away from compelling improvement and over intensification of clamor in the picture.

Ooi et al., [39] in 2010 presented clipping predicated Quadrants Dynamic Histogram evening out (QDHE), which divides the histogram into four sub-histograms predicated on the average of the information picture. From that point, the resultant sub-histograms are cut by mean of power event of the information picture before beginning element reach is appointed to each one sub-histogram and are balanced exclusively. QDHE is the most capable system to concentrate the subtle elements of the low difference pictures.

Liang et al., [40] in 2012, proposed Double Plateaus Histogram Equalization (DPHE) for infrared picture improvement. In this procedure, upper and lower edge qualities could be computed by examining nearby greatest and soothsaying least as interim and be redesigned incredible time. The estimation of upper edge is situated to be $20-30 \%$ of the pixels altogether, while the lower limit quality is situated to be $5-10 \%$ of it. The upper edge is utilized as a part of the calculation for dithering over-improvement of foundation commotion with commonplace grey levels, and the lower limit is situated for bulwarking definite data with fewer pixels from being connected.

Bi-HE routines improve the picture differentiate essentially and may protect the blaze to some degree, be that as it may it presents undesirable antiques. Disintegration of picture histogram into a few sub-histograms, Multi-HE techniques indicated exceeding expectation brilliance protection and block prelude of undesirable relics yet may not essentially upgrade the difference. CHE strategies give well brightness protection without any relics by cutting the histograms using limit values and are not demonstrated considerable difference upgrade. Routines like GC-CHE yielded the measure of difference in controlling clamor and for protecting the immaculate power level.

\section{IMAGE QUALITY MEASUREMENT TOOLS}

This segment portrays the Image quality measurement devices used to judge the capacity of the upgrade strategies to keep up the mean splendor conservation and difference improvement.

\subsection{Absolute Mean Brightness Error (AMBE)}

This is a target estimation which is proposed with the thought to rate the execution in protecting the perfect brightness. Total Mean Brightness Error (AMBE) is characterized as irrefutably the distinction between the information mean and the yield gray level of pictures [20,21].

$\mathrm{AMBE}=|\mathrm{E}[\mathrm{Y}]-\mathrm{E}[\mathrm{X}]|$

Where, $E[y]$ and $E[x]$ are the mean estimations of new and unique light black level of picture individually. Bring down the estimation of AMBE parameter better is the brilliance conservation of the picture. Eq (6) demonstrates that is intended to identify distortions-excessive shine changes [22].

\subsection{Tenengrad}

Contrast either increases or decreases by abruptly hanging of the slant of gray levels in the pictures. Slope is the incline between grey level pixels to distinguish the picture edge to confirm the picture quality. Tenengrad word is ascertained from slope of all picture pixels and it is focused around angle gradient magnitude maximization [43]. The gradient is computed as

$\mathrm{S}(\mathrm{x}, \mathrm{y})=\sqrt{\left(\mathrm{i}_{\mathrm{x}} * \mathrm{I}(\mathrm{x}, \mathrm{y})\right)^{2}+\left(\mathrm{i}_{\mathrm{y}} * \mathrm{I}(\mathrm{x}, \mathrm{y})\right)^{2}}$

Where * denotes convolution and $\mathrm{I}(\mathrm{x}, \mathrm{y})$ is the Enhanced Image.

The Tenengrad parameter is calculated as-

Tenengrad $=\sum_{\mathrm{x}} \sum_{\mathrm{y}} \mathrm{S}(\mathrm{x}, \mathrm{y})^{2}$

This parameter is used to confirm the improvement of the picture contrast. The higher estimation of this parameter connotes great sharpness and visibility.

\subsection{Peak Signal to Noise Ratio (PSNR)}

PSNR is characterized through Mean Squared Error parameter (MSE). This is utilized to gauge the nature of remaking by deciphering the encoded rendition of the source picture. PSNR is expressed as Logarithmic decibels (dB).

The Mean Squared Error is calculated as-

$\operatorname{MSE}=\frac{1}{\mathrm{MN}} \sum_{0}^{\mathrm{M}-1} \sum_{0}^{\mathrm{N}-1}\|\mathrm{X}(\mathrm{i}, \mathrm{j})-\mathrm{Y}(\mathrm{i}, \mathrm{j})\|^{2}$

Where, $X(i, j)$ is the source image and $Y(i, j)$ is the reconstructed image. Both source image and reconstructed image have $\mathrm{M}$ by $\mathrm{N}$ pixels.

Errors are computed on luminance signal so, $\mathrm{X}(\mathrm{i}, \mathrm{j})$ range from black (0) to white (255) [27].

The PSNR is defined as-

PSNR $=20 \cdot \log _{10}\left(\frac{\operatorname{Max}(\mathrm{Y}(\mathrm{i}, \mathrm{j}))}{\sqrt{\mathrm{MSE}}}\right)$

Greater is the value of PSNR, better will be the contrast enhancement in the image.

\section{DESIGN AND IMPLEMENTATION}

Segmentation divides a picture into unique districts containing every pixel with homogeneous traits. To be vital and utilizable for picture investigation and translation, the areas ought to energetically identify with delineated protests or gimmicks of premium. Weighty division is the first venture from low-level picture preparing to change a grayscale or shade picture into one or more different pictures to the abnormal state picture portrayal regarding gimmicks, articles, and scenes. The thriving of picture examination relies on upon unwavering quality of division, yet an exact parceling of a picture is by and large an issue.

The image division in this paper is carried out through the opening-by-reproduction. Performing morphological reproduction, using the dissolved picture as the marker and the perfect picture as the cover, is called "opening by reconstruction." In morphological opening, disintegration commonly edited compositions little protests, and the ensuing widening slopes to recover the state of the questions that remain. Notwithstanding, the exactness of this instauration relies on upon the homogeneous trait between the shapes and the organizing component. Opening by reconstruction, remodels the immaculate status of the questions that stay after disintegration. The opening by reconstruction of a picture $G$ using organizing component B is characterized as RG (GOB). The data flow of the proposed tool is shown in the following figure 6 


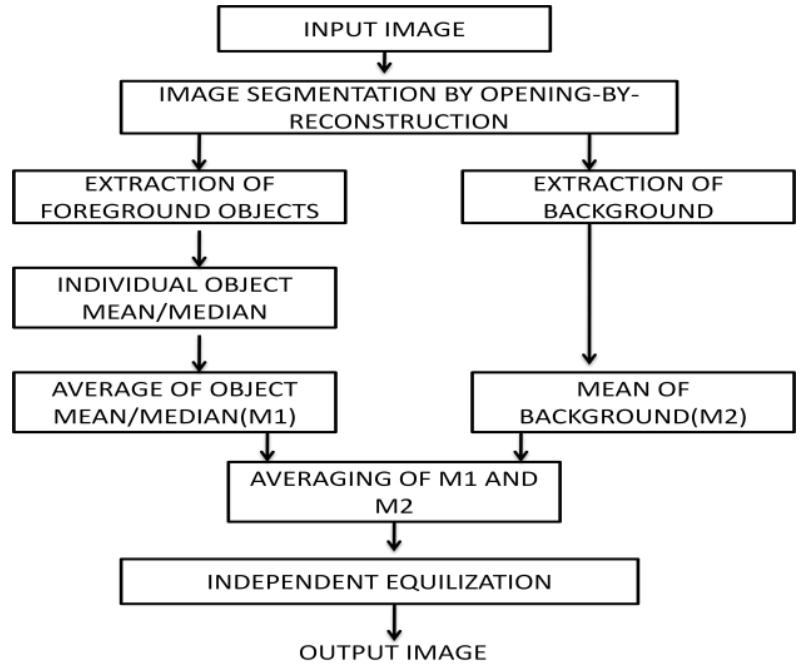

Figure 6 Steps of enhancement and Separation of foreground and background of the image

In consonance with the above dialogue, I assembled a pervasive structure for Image Enhancement. Figure 7 delineates the structure comprising of emulating four steps:

I. Image Selection

II. Image Segmentation (Extraction of foreground and background objects)

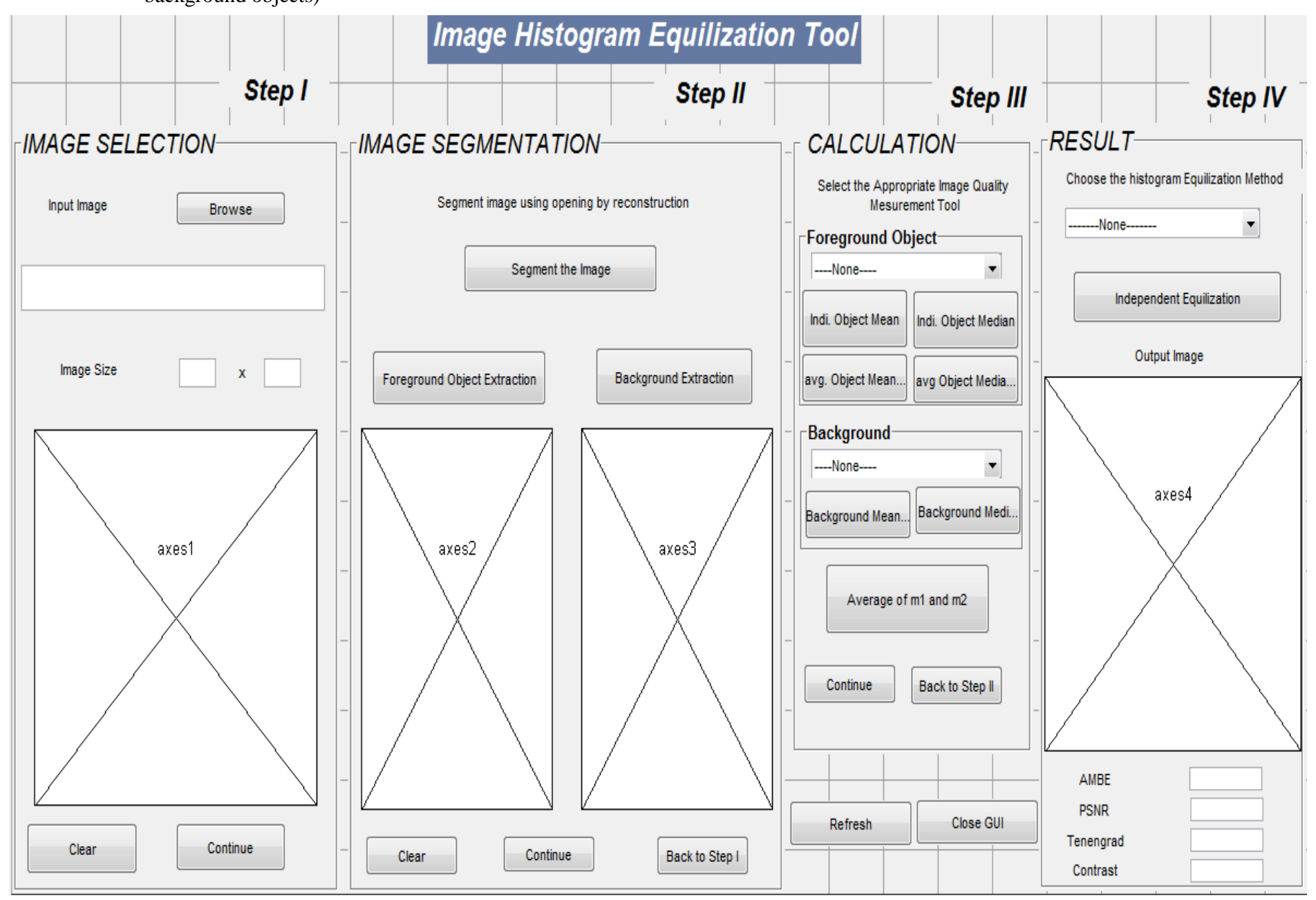

\section{Calculation of Mean/Median and}

IV. Choice of histogram equalization methods with Image quality measurements and Result

Histogram Equalization Techniques and Image quality Measurement apparatuses effectively talked about in the past segment 2 and 3 individually.

Above all else, the picture is chosen and this picture ought to be a light black scale picture. In the event that the picture is a colored picture, then this picture is changed over to the ash scale picture having size of $256 \times 256$. That is, the picture has 256 columns and 256 sections.

In the second step, the picture is sectioned using the morphological operation opening-by-reproduction to concentrate the forefront and foundation objects from the picture. Opening is disintegration trailed by expansion. Along these lines, opening-by-recreation is disintegration trailed by morphological reproduction. To do this, first figure the opening utilizing imopen () and after that process the openingby-remaking utilizing imerode () and imreconstruct (). To concentrate the foundation pixels edge operation is utilized as a part of which dim pixels have a place with the foundation. Here, the foundation is given the look of "meager" by processing the "skeleton by impact zones" of the forefront. This could be possible, by figuring the watershed change of the separation change, and after that searching for the watershed edge lines of the result.

Figure 7 Image Histogram Equalization Tool 
In the third step, calculation (Mean/Median relying on the client choice) is carried out on the closer view and foundation pixels. Last step uses the histogram choice technique. The client needs to choose the suitable picture histogram strategy and the yield is the upgraded picture. Relating picture quality measure qualities are likewise indicated on the upgraded picture toward the end of the apparatus. By utilizing this instrument we can strive for diverse relative investigation on the premise of histogram adjustment methods and picture quality measures. The accompanying area talks about the result.

\section{EXPERIMENTAL RESULTS AND DISCUSSION}

Numerous pictures are handled to demonstrate the execution of above examined histogram adjustment procedures. This area displays the relative investigation of the histogram evening out focused around picture contrast systems, including histogram equalization (HE) depicted in the point of reference areas. For relative examination, the histogram evening out techniques are contrasted and distinctive test pictures. Bi-histogram adjustment systems, Multi-histogram evening out routines and Clipped-histogram balance strategy calculation is readied using the MATLAB device MATLAB R2012a. After that, the systems are likewise contrasted and the picture quality estimation instruments, for example, AMBE, PSNR and Tenengrad. Aftereffect of test pictures are demonstrated in figure 9, figure 10, figure 11 and the picture quality estimation, for example, AMBE, PSNR, Tenengrad qualities are arranged in table I, table II and table III.

By contrasting test pictures, it is discovered that, the AMBE values for shine Preservation, Dynamic Histogram Equalization (BPDHE) and Gain-Controllable Clipped Histogram Equalization (GC-CHE) strategies are exceptionally proximate to zero as demonstrated in Table 1. Thus, these techniques have indicated astounding brilliance protection than different strategies. Higher PSNR estimations of these strategies are speaking to great difference improvement. The yield pictures (Tire and Match_Stick) of these techniques are extremely pellucid from different pictures as demonstrated in figure 9, figure 10 and figure 11 .
Bi-Histogram Equalization Plateau Limit (BHEPL) strategy excessively demonstrated great shine protection (low AMBE worth) like 0.4424 for Match_Stick picture and 0.5167 for base left picture. The brilliant pictures like Face, Hurricane and Lenna has demonstrated AMBE values as 10.1918, 18.4798, and 10.5876 individually and the improvement of complexity (high PSNR value) additionally great in all most all test pictures as indicated in Table 1 and Table 2.

Dynamic Histogram Equalization (DHE) method is a larger number of stronger than AMBE (Table I) assigning less shine protection and less estimations of PSNR (Table II) with poor difference improvement. From Figures 9(i) and 10(i), the yield pictures (Tire and Match_stick) of DHE technique are not fine and presented obnoxious commotion. BPDHE, the expansion of DHE has indicated better splendor protection and differentiation upgrade. The AMBE estimations of BBDHE are extremely proximate to GC-CHE and indistinguishably equivalent to zero as indicated in Table 1 . From the Figures 9(j), 10(j) and 11(j), it is pellucid that yield pictures of BPDHE are superior to DHE yield pictures. Recursively Dissevered and Weighted Histogram Equalization (RSWHE) has demonstrated poor shine conservation and better difference upgrade as indicated in Figures 9(h), 10(h) and 11(h).

Recursive Sub-Image Histogram Equalization (RSIHE) strategy had demonstrated well, brightness protection for pictures Bottom_Left, Cameraman, Face, Hurricane and Lenna. The yield picture of RSIHE, the Tire has over improved and the data are muddled at the base share of the picture as indicated in Figure 9(g). For picture Match_Stick and Couple, RSIHE presented maddening clamor and brought about a loss of data and obscured as indicated in Figure 10(g) and $11(\mathrm{~g})$.

Recursive Mean-Separate Histogram Equalization (RMSHE) technique has demonstrated splendor safeguarding for the dim pictures like base left and self-assertive matches. RMSHE has indicated over-upgrade alongside unpalatable clamor as demonstrated in Figures 9(f), 10(f) and 11(f).

\subsection{Analysis Results Table}

Table 1AMBE Utilized for Brightness Preservation

\begin{tabular}{|c|c|c|c|c|c|c|c|c|c|c|c|}
\hline \multirow{2}{*}{$\begin{array}{l}\text { Method } \\
\text { Images }\end{array}$} & \multicolumn{4}{|c|}{ Bi-HE Methods } & \multicolumn{5}{|c|}{ Multi-HE Methods } & \multicolumn{2}{|c|}{ CHE Methods } \\
\hline & $\begin{array}{l}\mathbf{H E} \\
\end{array}$ & BBHE & DSIHE & MMBEBHE & RMSHE & RSIHE & RSWHE & DHE & BPDHE & BHEPL & GC-CHE \\
\hline Tire & 71.8829 & 11.5840 & 11.5810 & 7.8103 & 11.5840 & 13.2906 & 87.3162 & 12.2210 & 0.0173 & 11.3142 & 0.0205 \\
\hline Match_Stick & 23.6291 & 10.3016 & 10.3016 & 8.5148 & 8.1032 & 13.1470 & 94.6552 & 56.2338 & 0.0169 & 0.5120 & 0.2190 \\
\hline Bottom_left & 87.1431 & 1.3980 & 1.3092 & 0.7738 & 1.3804 & 0.1235 & 31.6587 & 93.3186 & 0.0084 & 0.5337 & 0.0151 \\
\hline Cameraman & 16.3153 & 6.2992 & 6.2992 & 6.354 & 52.4698 & 0.5391 & 80.5401 & 4.3782 & 0.0186 & 33.7843 & 0.1621 \\
\hline Face & 34.6145 & 5.3152 & 5.3152 & 5.7532 & 14.3160 & 3.7767 & 80.1354 & 59.2653 & 0.0064 & 11.1519 & 0.1483 \\
\hline Lenna & 25.6653 & 12.4589 & 12.4589 & 10.8831 & 31.6453 & 4.7751 & 95.8513 & 56.0132 & 0.0423 & 18.4420 & 0.1024 \\
\hline Hurricane & 44.7321 & 11.5234 & 11.5234 & 2.0036 & 33.7582 & 4.3321 & 85.8391 & 24.1734 & 0.0176 & 23.1731 & 0.1604 \\
\hline Pirate & 19.1620 & 10.6321 & 10.6321 & 7.4165 & 28.6210 & 11.6106 & 89.1368 & 4.7125 & 0.0156 & 17.5788 & 0.1762 \\
\hline Boy & 69.4536 & 10.3478 & 10.3478 & 9.5611 & 10.5227 & 13.4299 & 84.3998 & 15.4476 & 0.0267 & 16.2216 & 0.0415 \\
\hline Hands & 73.6550 & 13.4277 & 13.4277 & 7.3412 & 7.2556 & 15.3889 & 85.7745 & 13.4550 & 0.0344 & 14.4490 & 0.1341 \\
\hline Couple & 21.3468 & 11.2086 & 11.2086 & 10.2158 & 6.2887 & 15.3269 & 91.7843 & 54.7632 & 0.0178 & 0.6470 & 0.2600 \\
\hline
\end{tabular}


Table 2 PSNR Utilized for Contrast Enhancement

\begin{tabular}{|c|c|c|c|c|c|c|c|c|c|c|c|}
\hline \multirow{2}{*}{$\begin{array}{l}\text { Method } \\
\text { Images }\end{array}$} & \multicolumn{4}{|c|}{ Bi-HE Methods } & \multicolumn{5}{|c|}{ Multi-HE Methods } & \multicolumn{2}{|c|}{ CHE Methods } \\
\hline & HE & BBHE & DSIHE & MMBEBHE & RMSHE & RSIHE & RSWHE & DHE & BPDHE & BHEPL & GC-CHE \\
\hline Tire & 22.2467 & 24.8767 & 24.8767 & 25.5001 & 24.8621 & 24.3124 & 21.1340 & 11.5587 & 26.6243 & 25.8445 & 59.8075 \\
\hline Match_Stick & 27.6395 & 26.4751 & 26.4751 & 29.4850 & 27.5728 & 25.9265 & 23.1410 & 10.9587 & 27.7823 & 40.3751 & 33.1420 \\
\hline Bottom_left & 23.5698 & 40.3245 & 40.3245 & 41.7213 & 30.4523 & 42.4580 & 23.4532 & 8.4090 & 21.5233 & 48.7321 & 44.3330 \\
\hline Cameraman & 27.5622 & 11.2800 & 11.2800 & 12.2332 & 11.2800 & 12.2385 & 23.1561 & 6.1521 & 28.8771 & 26.7921 & 43.4476 \\
\hline Face & 26.1356 & 15.0530 & 21.4429 & 26.0104 & 27.0672 & 30.0037 & 22.1893 & 10.0106 & 29.0130 & 28.0146 & 42.0232 \\
\hline Lenna & 26.6148 & 14.6210 & 14.6210 & 15.3154 & 14.0628 & 14.3546 & 25.2153 & 12.520 & 31.7734 & 27.5819 & 39.3859 \\
\hline Hurricane & 24.3319 & 13.4843 & 13.4843 & 15.0233 & 15.9076 & 14.0395 & 23.0643 & 14.9032 & 30.0831 & 26.0278 & 35.8501 \\
\hline Pirate & 26.1142 & 17.0327 & 15.4435 & 15.6532 & 15.9873 & 15.7298 & 22.54510 & 13.8894 & 31.3500 & 26.9257 & 37.5781 \\
\hline Boy & 21.0028 & 19.6276 & 18.9265 & 15.1255 & 23.7784 & 17.3460 & 22.7810 & 14.7221 & 26.5997 & 25.8152 & 32.6588 \\
\hline Hands & 23.3487 & 20.0351 & 18.2410 & 21.7621 & 30.5543 & 24.5449 & 25.4113 & 15.4428 & 28.5230 & 28.4998 & 32.1378 \\
\hline Couple & 24.3880 & 15.0291 & 16.9242 & 23.3921 & 21.9040 & 24.9072 & 23.0858 & 18.041 & 28.9848 & 26.4867 & 37.3217 \\
\hline
\end{tabular}

Table 3 Tenengrad utilized for the enhancement of Image Contrast

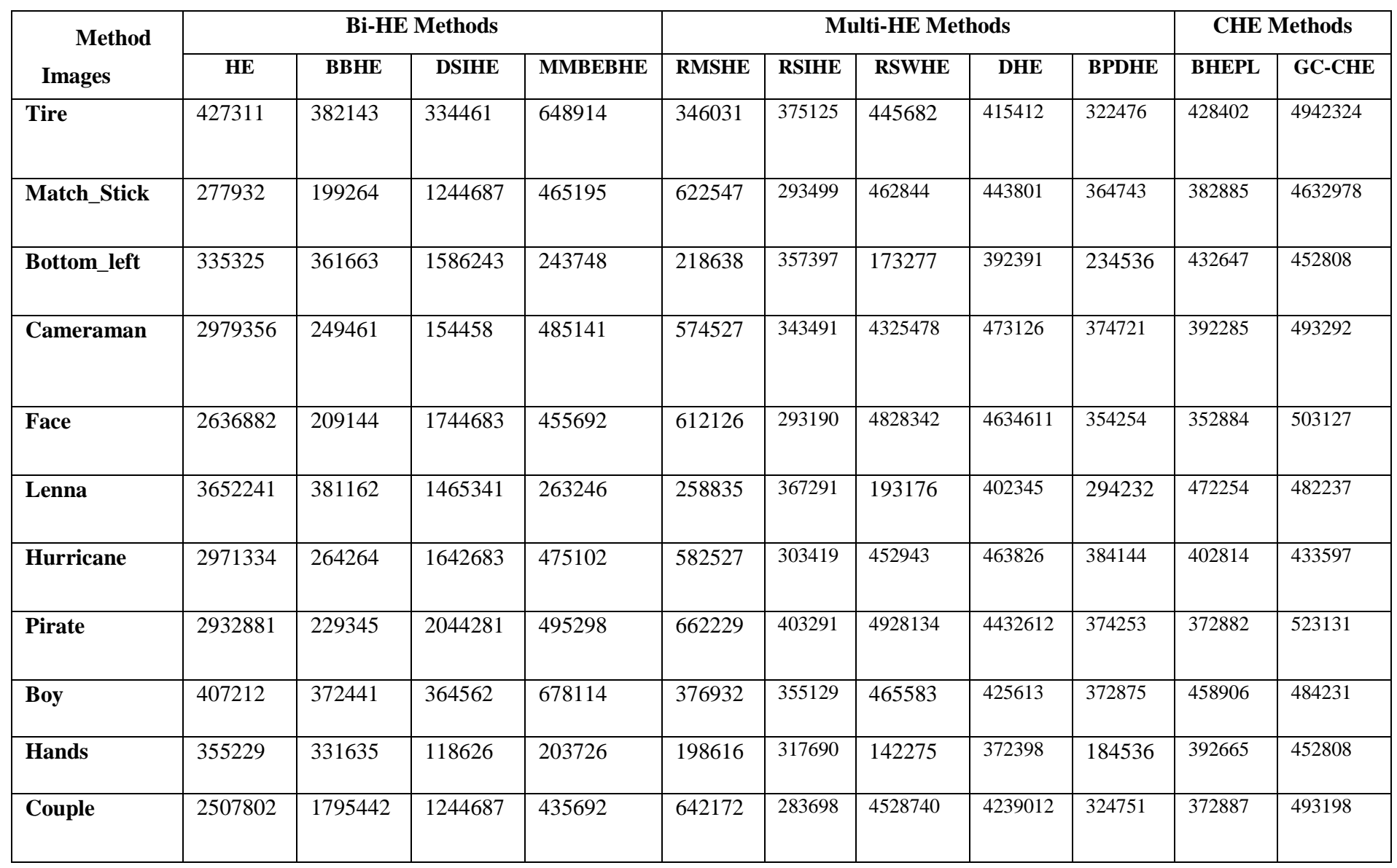




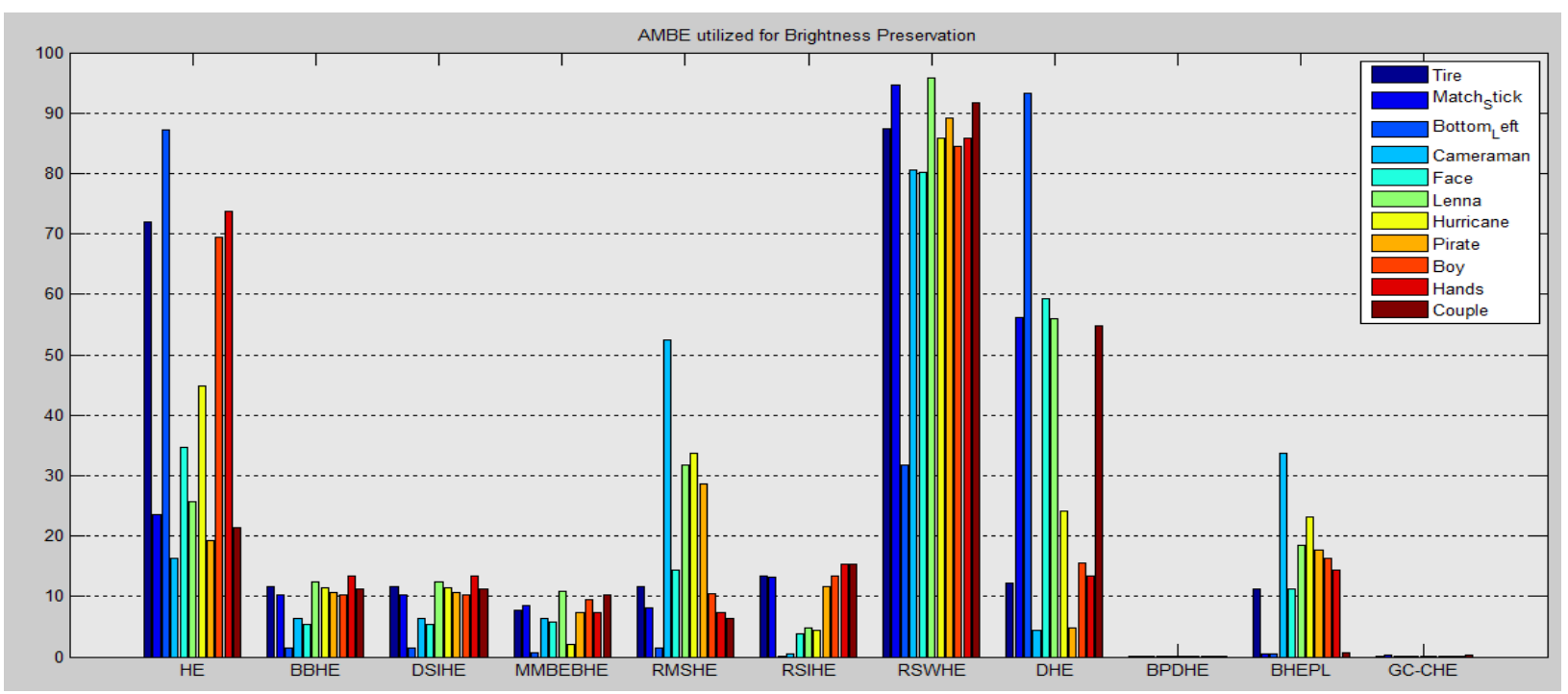

(a)

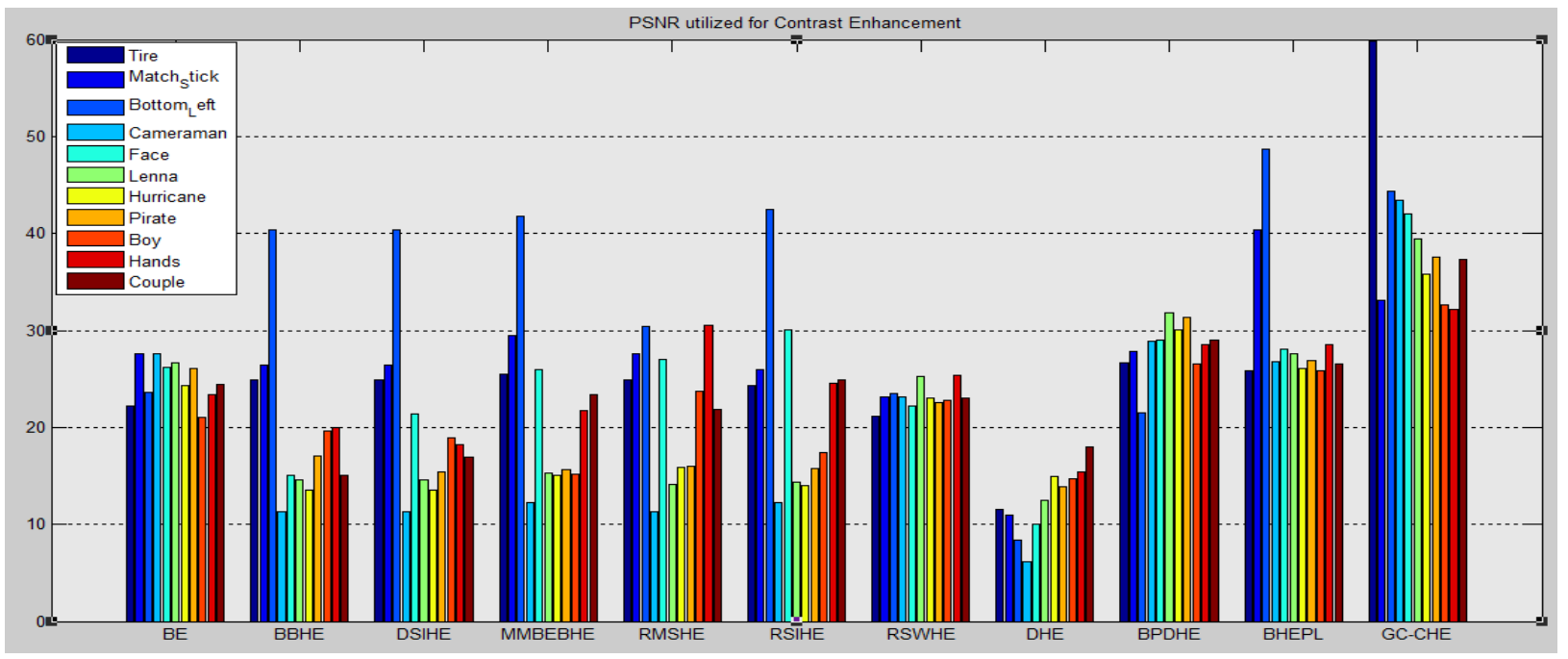

(b)

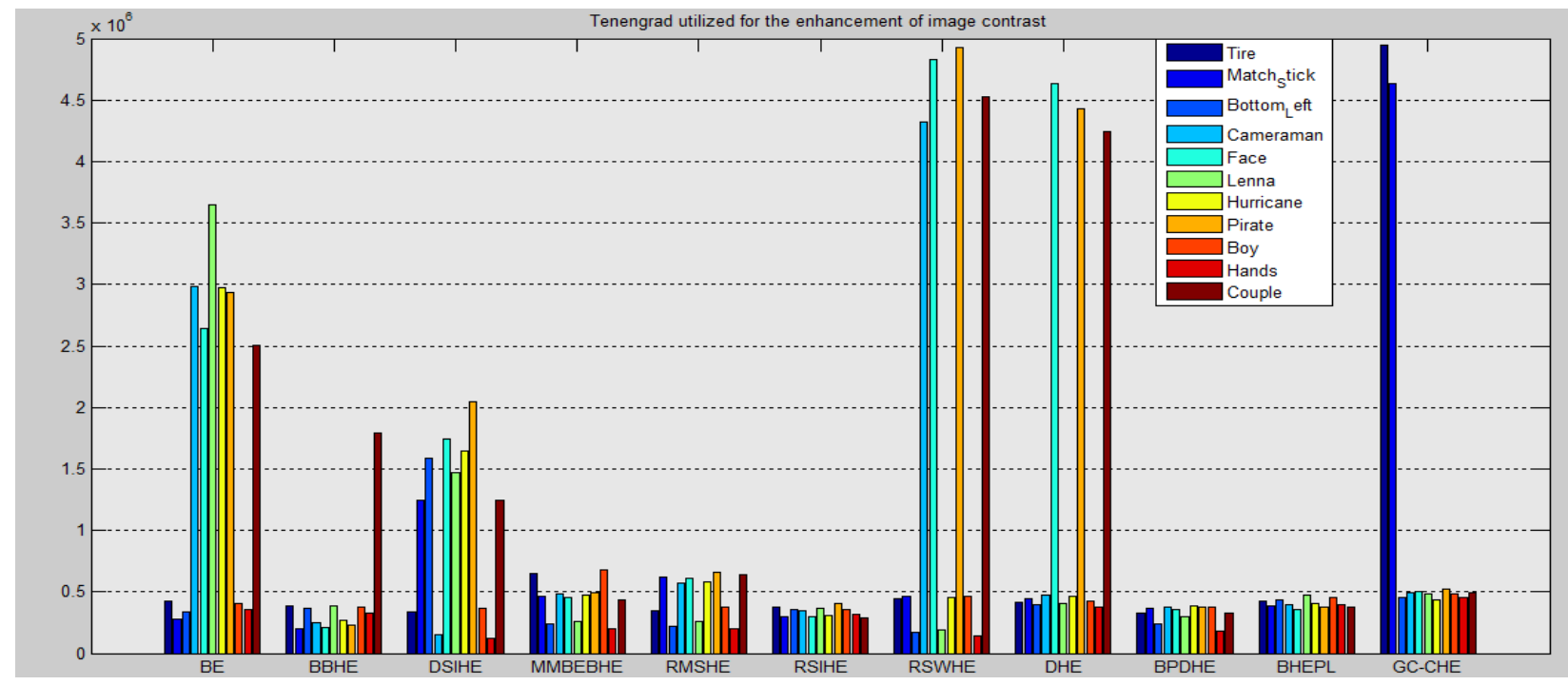

(c)

Figure 8 (a) Shows chart of AMBE for different HE Methods (b) Shows chart of PSNR for different HE Methods (c) Shows chart of Tenengrad for different HE Methods 
In Minimum Mean Brightness Error (AMBE) Bi-Histogram Equalization (MMBEBHE) technique had shown better brightness preservation. There is no much variation, in contrast enhancement as compared to DSIHE and BBHE. MMBEBHE is an elongated method of BBHE and showed a better contrast enhancement and brightness preservation than BBHE. However, it is not controlled over-enhancements as shown in Figure 9(e) and revealed less brightness preservation for bright images from Table 1 .

Brightness Preserving Bi-Histogram Equalization (BBHE) techniques and Dualistic Sub-Image Histogram Equalization (DSIHE) has shown all most same contrast enhancement and brightness preservation for all test images. More value of AMBE and over-enhancement are shown by BBHE and DSIHE as shown in Figures 9(c), 9(d), 10(c) and 10(d).

\subsection{Inspection of Visual Quality}
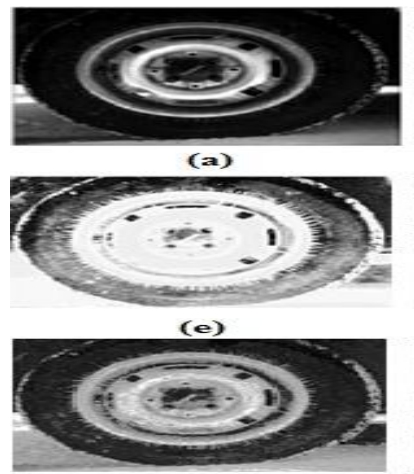

(i)

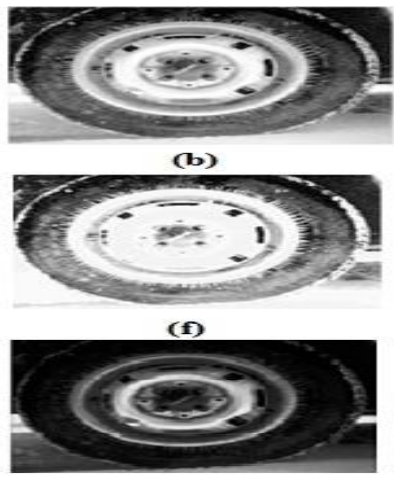

(i)

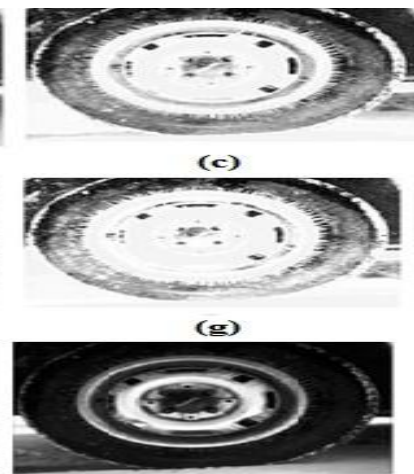

(lk)

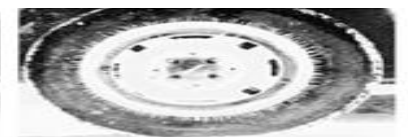

(d)

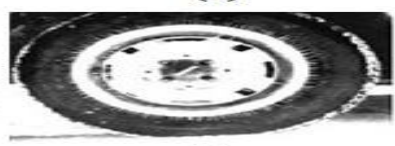

(h)

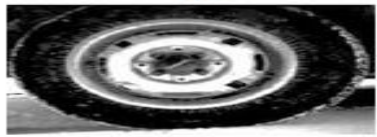

(1)

Figure 9 Comparison of Performance with Tire Image

(a) Original Image (b). Histogram Equalization, (c).Brightness Preserving Bi-Histogram Equalization (BBHE), (d). Dualistic Sub-Image Histogram Equalization (DSIHE), (e). Minimum Mean Brightness Error Bi-Histogram Equalization (MMBEBHE), (f). Recursive Mean-Separate Histogram Equalization (RMSHE) (g). Recursive SubImage Histogram Equalization (RSIHE), (h). Recursively Separated and Weighted Histogram Equalization (RSWHE), (i). Dynamic Histogram Equalization (DHE), (j). Brightness Preserving Dynamic Histogram Equalization (BPDHE), (k). Bi-Histogram Equalization with Plateau Limit (BHEPL) and (l). Gain-Controllable Clipped Histogram Equalization (GC-CHE)

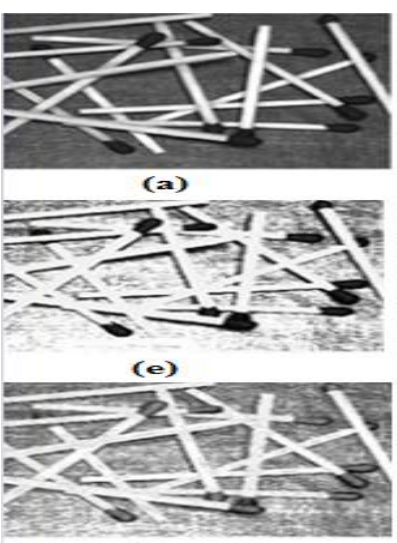

(i)

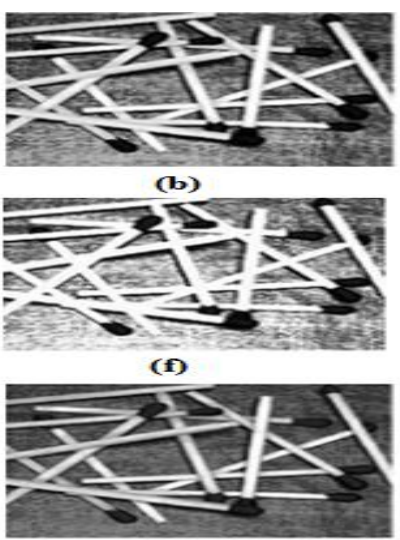

(i)

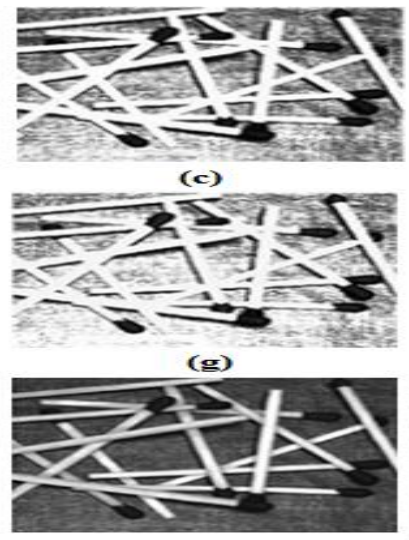

(k))

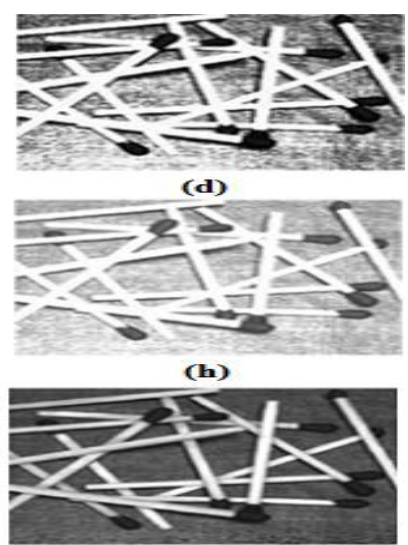

(1)

Figure 10 Comparison of Performance with Match_Stick

(a) Original Image (b). Histogram Equalization, (c).Brightness Preserving Bi-Histogram Equalization (BBHE), (d). Dualistic Sub-Image Histogram Equalization (DSIHE), (e). Minimum Mean Brightness Error Bi-Histogram Equalization (MMBEBHE), (f). Recursive Mean-Separate Histogram Equalization (RMSHE) (g). Recursive SubImage Histogram Equalization (RSIHE), (h). Recursively Separated and Weighted Histogram Equalization (RSWHE), (i). Dynamic Histogram Equalization (DHE), (j). Brightness Preserving Dynamic Histogram Equalization (BPDHE), (k). Bi-Histogram Equalization with Plateau Limit (BHEPL) and (l). Gain-Controllable Clipped Histogram Equalization (GC-CHE) 


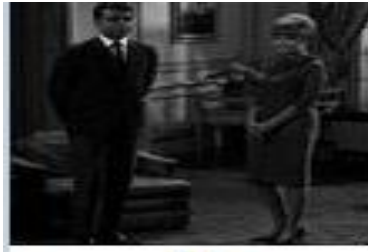

(a)

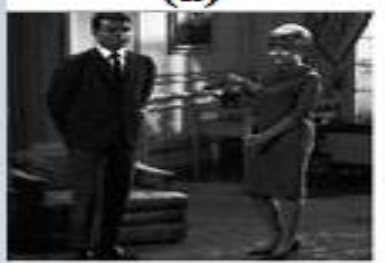

(e)

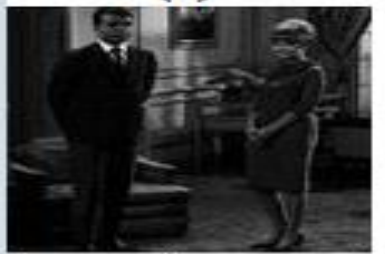

(i)

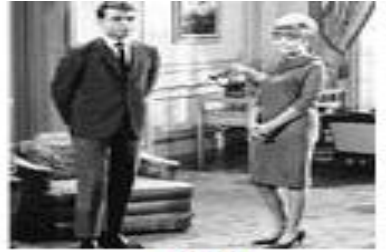

(b)

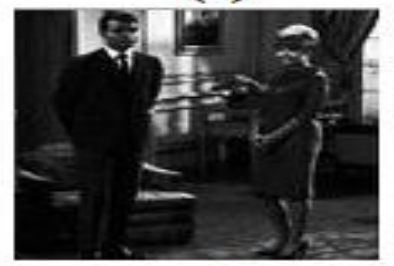

(f)

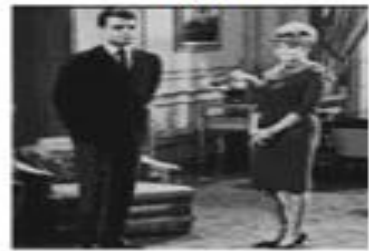

(j)

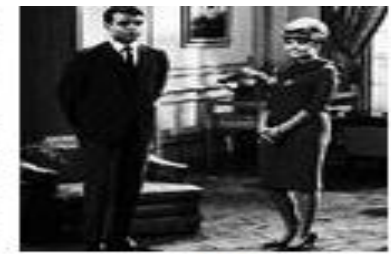

(c)

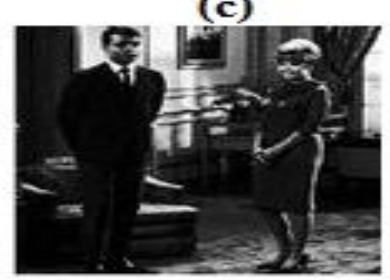

(g)

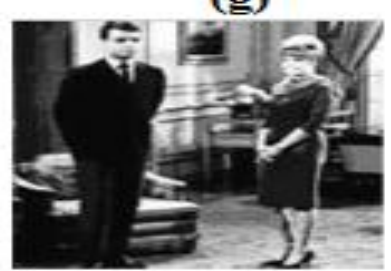

(k)

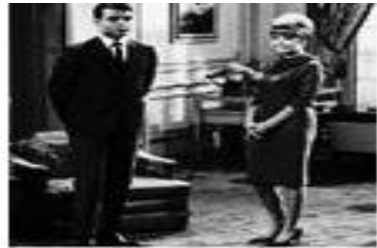

(d)

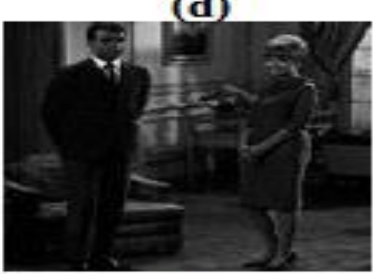

(h)

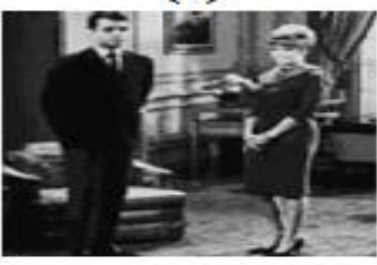

(I)

Figure 11 Comparison of Performance with Couple Image

(a) Original Image (b). Histogram Equalization, (c).Brightness Preserving Bi-Histogram Equalization (BBHE), (d). Dualistic Sub-Image Histogram Equalization (DSIHE), (e). Minimum Mean Brightness Error Bi-Histogram Equalization (MMBEBHE), (f). Recursive Mean-Separate Histogram Equalization (RMSHE) (g). Recursive SubImage Histogram Equalization (RSIHE), (h). Recursively Separated and Weighted Histogram Equalization (RSWHE), (i). Dynamic Histogram Equalization (DHE), (j). Brightness Preserving Dynamic Histogram Equalization (BPDHE), (k). Bi-Histogram Equalization with Plateau Limit (BHEPL) and (l). Gain-Controllable Clipped Histogram Equalization (GC-CHE)

In the present situation, it can effortlessly be comprehended the objective of Enhancing Images. Whether it's a visual for an architect, a craftsman: who needs a misshaped and unattractive picture? Indeed a viewer of Cricket Match or a motion picture lover in a theater needs perfect looking pictures. In writing such a large number of calculations are proposed for picture contrast.

The customary strategies for picture improvement have hindrances, for example, these routines simply experience the ill effects of the fractional good faith. That is, they have, the less correct at the regulation of its speed and the course.

These routines can't work out the issues of dispersing and improvement.

The point of this work is to use the speedy improvement method of upgrade of pictures in order to decrease the preparing time and get effective and exact results.

\section{CONCLUSION}

The present paper gives the GUI instrument for picture upgrade methods through histogram leveling systems for splendor safeguarding and picture contrast improvement. Bihistogram balance systems, Multi-histogram leveling routines and Clipped histogram evening out techniques are contrasted and picture quality estimation apparatuses like AMBE, PSNR and Tenengrad. All the procedures have surmounted the disadvantages of histogram leveling and have demonstrated better brilliance conservation and differentiation upgrade than HE. For strategies like BBHE, DSIHE, MMBEBHE, RMSHE, RSIHE and RSWHE, the differentiation of the pictures is enhanced, yet the issue of the force immersion happens in a few locales of the picture also and furthermore displayed invigorated intensification of commotion in the yield picture. All these systems show luminosity safeguarding, yet demonstrated a lesser brilliance protection for radiant pictures like a Pirate and Lenna. MMBEBHE, the augmentation system for BBHE has indicated better brilliance conservation. Anyhow, it couldn't control over-upgrade of the picture. Because of over-upgrade in MMBEBHE, RMSHE and RSIHE, there is a data misfortune in the yield picture. The RSIHE system as furthermore had demonstrated all the more over-improvement than MMBEBHE and RMSHE. RSWHE and DHE routines have demonstrated great brightness protection and also a controlled over-improvement, yet presented angering clamor in the yield picture. BHEPL strategy has additionally demonstrated great splendor conservation aside from radiant pictures like Cameraman, Face, Hurricane, Pirate and Lenna. BPDHE system has given less estimation of AMBE, and is basically zero (0.0067 to 0.0911) assigning great luster safeguarding and high estimations of PSNR shows better complexity upgrade. GCCHE system has likewise indicated less estimations of AMBE (0 to 0.1827) and high estimations of PSNR (34.1527 to 61.6174). The yield pictures of BPDHE and GC-CHE are extremely pellucid without any clamor and there is no loss of data. BPDHE and GC-CHE strategies are more harmonious for purchase electronic items, where safeguarding the immaculate luster is fundamental. Future applications include photographs got from satellite interchanges. Other application fields are Medical field-Meteor portrayals, X-Rays, climate data, Revelations of Geostationary bodies, the law authorizes that incorporates observation cam handling, finger preparing, and Science- improving electron magnifying instrument picture for coherence. 


\section{REFERENCES}

[1] R. C. Gonzalez and R. E. Woods, " Digital Image Processing", vol. 2nd edition, Prentice Hall, 2002.

[2] S. C. Pei, Y. C. Zeng and C. H. Chang, "Virtual restoration of ancient Chinese paintings using color contrast enhancement and lacuna texture synthesis", IEEE Transaction on. Image Processing, vol. 13, 2004, pp. 416-429.

[3] A. Wahad, S. H. Chin and E. C. Tan, "Novel approach to automated fingerprint recognition", IEEE Proceedings on Vision, Image and Signal Processing, vol. 145, 1998, pp. 160-166.

[4] A. Torre, A. M. Peinado, J. C. Segura, J. L. PerezCordoba, M. C. Benitez and A. J. Rubio, "Histogram equalization of speech representation for robust speech recognition", IEEE Tranaction on. Speech Audio Processing, vol. 13, 2005, pp. 355-366.

[5] S. M. Pizer, "The medical image display and analysis group at the University of North Carolina: Reminiscences and philosophy", IEEE Transaction on Medical Image, vol. 22, 2003, pp. 2-10.

[6] A. H. Ooi and N. A. Mat Isa, "Adaptive Contrast Enhancement Methods with Brightness Preserving", IEEE Transactions on Consumer Electronics, vol. 56, no. 4, 2010, pp. 2543-2551.

[7] Y. T. Kim, "Contrast Enhancement Using Brightness Preserving Bi-Histogram Equation”, IEEE Transactions on Consumer Electronics, vol. 43, no. 1, February 1997, pp. 1-8.

[8] R. C. Gonzalez and R. E. Woods, "Digital Image Processing", 2nd edition, MA. Addison-Wesley, 1992, pp. $85-103$.

[9] J. Zimmerman, S. Pizer, E. Staab, E. Perry, W. McCartney and B. Brenton, "Evaluation of the effectiveness of adaptive histogram equalization for contrast enhancement", IEEE Transaction on Medical Imaging, 1988, pp. 304-312.

[10] T. K. Kim, J. K. Paik and B. S. Kang, "Contrast enhancement system using spatially adaptive histogram equalization with temporal filtering", IEEE Transaction on Consumer Electronics, vol. 44, no. 1, 1998, pp. 82-86.

[11] R. C. Gonzalez and P. Wints, "Digital Image Processing", 2nd edition, Ed., Massachusetts: AddisonWesley Publishing Co., Reading, 1987.

[12] N. Sengee and H. K. Choi, "Brightness preserving weight clustering histogram equalization", IEEE Transactions on Consumer Electronics, vol. 54, no. 3, 2008, pp. 1329-1337.

[13] T. Kim and J. Paik, "Adaptive Contrast Enhancement Using Gain-Controllable Clipped Histogram Equalization", IEEE Transactions on Consumer Electronics, vol. 54, no. 4, November 2008, pp. 18031810 .

[14] Y. Li, W. Wang and D. Y. Yu, "Application of adaptive histogram equalization to X-ray chest image", Proceedings of the SPIE, vol. 2321, 1994, pp. 513-514.
[15] Q. Wang and R. K. Ward, "Fast image/video contrast enhancement based on weighted threshold histogram equalization", IEEE transactions on Consumer Electronics, vol. 53, no. 2, 2007, pp. 757-764.

[16] M. Kim and M. G. Chung, "Recursively Separated and Weighted Histogram Equalization for Brightness Preservation and Contrast Enhancement", IEEE Transactions on Consumer Electronics, vol. 54, no. 3, August 2008, pp. 1389-1397.

[17] Y. Wang, Q. Chen and B. Zhang, "Image Enhancement Based on Equal Area Dualistic Sub-Image Histogram Equalization Method", IEEE Transactions on Consumer Electronics, vol. 45, no. 1, 1999, pp. 68-75.

[18] S.-D. Chen and A. R. Ramli, "Minimum Mean Brightness Error Bi-Histogram Equalization in Contrast Enhancement", IEEE Transactions on Consumer Electronics, vol. 49, no. 4, November 2003, pp. 13101319.

[19] S.-D. Chen and A. R. Ramli, "Preserving brightness in histogram equalization based contrast enhancement techniques", Digital Signal Processing, vol. 14, 2004, pp 413-428.

[20] S.-D. Chen, "A new image quality measure for assessment of histogram equalization-based contrast enhancement technique", Digital Signal Processing, vol. 22, 2012, pp. 640-647.

[21] N. Sengee, A. Sengee and H. K. Choi, "Image Contrast Enhancement using Bi-Histogram Equalization with Neighborhood Metrics", IEEE Transactions on Consumer Electronics, vol. 56, no. 4, November 2010, pp. 2727-2734.

[22] A. Zuo, Q. Chen and X. Sui, "Range Limited BiHistogram Equalization for Image Contrast Enhancement", Optik, 2012.

[23] K. Wongsritong, K. Kittayaruasiriwat, F. Cheevasuvit, K. Deihan and A. Somboonkaew, "Contrast Enhancement using Multipeak Histogram Equalization with Brightness Preserving", IEEE Asia-Pacific Conference on Circuits and Systems, 1998.

[24] S.-D. Chen and A. R. Ramli, "Contrast Enhancement using Recursive Mean-Separate Histogram Equalization for Scalable Brightness Preservation”, IEEE Transactions on Consumer Electronics, vol. 49, no. 4, November 2003, pp. 1301-1309.

[25] K. S. Sim, C. P. Tso and Y. Y. Tan, "Recursive subimage histogram equalization applied to gray scale images", Pattern recognition Letters, vol. 28, 2007, pp. 1209-1221.

[26] M. A. A. Wadud, M. H. Kabir, M. A. A. Dewan and O. Chae, "A Dynamic Histogram Equalization for Image Contrast Enhancement", IEEE Transactions on Consumer Electronics, vol. 53, no. 2, May 2007, pp. 593600 .

[27] H. Ibrahim and N. S. Pik Kong, "Brightness Preserving Dynamic Histogram Equalization for Image Contrast Enhancement", IEEE Transactions on Consumer Electronics, vol. 53, no. 4, November 2007, pp. 1752 1758. 
[28] A. Menotti, L. Najman, J. Facon and A. D. A. Araujo, "Multi-Histogram Equalization Methods for Contrast Enhancement and Brightness Preserving", IEEE Transactions on Consumer Electronics, vol. 53, no. 3, August 2007, pp. 1186-1194.

[29] N. Sengee and H. K. Choi, "Brightness preserving weight clustering histogram equalization", IEEE Transactions on Consumer Electronics, vol. 54, no. 3, 2008, pp. 1329-1337.

[30] M. A. A. Wadud, M. H. Kabir and O. Chae, "A Spatially Controlled Histogram Equalization for Image Enhancement", 23rd International Symposium on Computer and Information Sciences, ISCIS '08, 2008.

[31] A. Sheet, H. Garud, A. Suveer, M. Mahadevappa and J. Chatterjee, "Brightness Preserving Dynamic Fuzzy Histogram Equalization", IEEE Transactions on Consumer Electronics, vol. 56, no. 4, November 2010, pp. 2475-2480.

[32] M. Khan, E. Khan, and Z. A. Abbasi, "Weighted average multi segment histogram equalization for brightness preserving contrast enhancement", IEEE International Conference on Signal Processing, Computer and Control, ISPCC, 2012.

[33] S. Yang, J. H. Oh and Y. Park, "Contrast Enhancement using Histogram Equalization with Bin Underflow and Bin Overflow", International Conference on Image Processing ICIP-2003, vol. 1, September 2003, pp. 881884.

[34] B.-J. Wang, S.-q. Liu, Q. Li and H.-X. Zhou, "A realtime Contrast Enhancement Algorithm for Infrared Images based on Plateau Histogram", Infrared Physics \& Technology, vol. 48, 2006, pp. 77-82.

[35] S. P. K. Nicholas, H. Ibrahim, C. H. Ooi and D. C. J. Chieh, "Enhancement of Microscopic Images using Modified Self-Adaptive Plateau Histogram Equalization", IEEE-Computer Society-2009 International Conference on Computer Technology and Development, 2009.
[36] C. H. Ooi, N. S. Pik Kong and H. Ibrahim, "BiHistogram Equalization with a Plateau Limit for Digital Image Enhancement", IEEE Transactions on Consumer Electronics, vol. 55, no. 4, November 2009, pp. 2072 2080.

[37] C. H. Ooi and N. A. M. Isa, "Quadrants Dynamic Histogram Equalization for Contrast Enhancement", IEEE Transactions on Consumer Electronics, vol. 56, no. 4, 2010, pp. 2552-2559.

[38] K. Liang, Y. Ma, Y. Xie, B. Zhou and R. Wang, “A new adaptive contrast enhancement algorithm for infrared images based on double plateaus histogram equalization", Infrared Physics \& Technology, vol. 55, 2012, pp. 309-315.

[39] C.Chaudhary, M.K.Patil, "Review of image enhancement techniques using histogram equalization", International journal of application or innovation in engineering \& management, vol.2, no. 5, May 2013, pp. 343-349.

[40] S.Nimkar, S.Shrivastava and S.Varghese, "Contrast enhancement and brightness preservation using multidecomposition histogram equalization", International journal of signal and image processing, vol. 3, no. 4, June 2013, pp. 83-89.

[41] O. Patel, Y.P.S. Maravi and S. Sharma, "A comparative study of histogram equalization based image enhancement techniques for brightness preservation and contrast enhancement", International journal of signal and image processing, vol. 4, no. 5, October 2013, pp. $11-25$.

[42] A. S. Krishna, G.S Rao and M.Sravya, "Contrast enhancement techniques using histogram equalization methods on color images with poor lighting", International journal of computer science, engineering and applications, vol. 3, no. 5, August 2013, pp.15-24.

[43] N.Phanthuna, F. Cheevasuvit and S. Chitwong, "Contrast enhancement for minimum mean brightness error from histogram partitioning", Proceedings of American Society for Photogrammetry and Remote Sensing (ASPRS), Baltimore, Maryland, March 2013. 